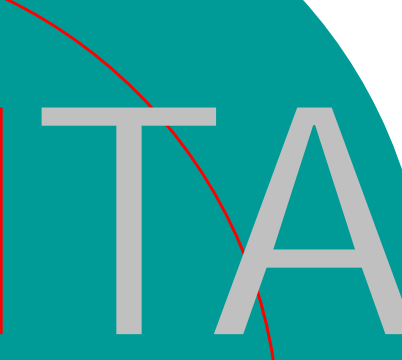

Health Technology Assessment

\section{ITA}

INSTITUT FÜR TECHNIKFOLGENABSCHÄTZUNG

Inhalt

\section{Stammzellenforschung: quo Vadis?}

Ist das Potenzial adulter Stammzellen schon ausgeschöpft?

\section{Hyperbare \\ Sauerstofftherapie/HBO}

Versorgung des Körpers mit Sauerstoff.

\section{Sakrale}

\section{Neurostimulation/SNS bei Inkontinenz}

Implantation eines

"Schrittmachers"

\section{Spasmolytika:}

\section{u. a. Botulinum toxin/Bt}

Behandlung von Spastik 3

\section{Alzheimer-Medikamente}

Donepezil, Rivastigmine,

Galantamine, Metrifonte 3

\section{HTA-Suchhilfe}

HTA-Linkliste auch als Rechercheseminar 4

\title{
STAMMZELLENFORSCHUNG: QUO VADIS?
}

\section{Ist das Potenzial adulter Stammzellen schon ausgeschöpft?}

Die klinische Forschung und Anwendung mit Stammzellen ist derzeit Inhalt zahlreicher klinischer, bioethischer und gesundheitspolitischer Diskussionen. Fast immer stehen die ethisch umstrittenen embryonalen Stammzellen im Zentrum. Die Diskussion ist von großen Erwartungen geprägt. Eine Bewertung der Möglichkeiten, Grenzen und des Nutzens der Anwendungen von ethisch nicht umstrittenen adulten Stammzellen wäre aber wichtig.

Im Zuge des bioethischen Diskurses zur Nutung embryonaler Stammzellen mehren sich die Wortmeldungen, dass das Potenzial adulter Stammzellen nicht ausgeschöpft ist und dass die Möglichkeiten adulter Stammzellen unterschätt werden.

Während adulte Stammzellen bereits determiniert sind, d. h. dem entnommenen Gewebe oder Organ entsprechend vorbestimmt sind, sind embryonale Stammzellen "toti- bzw. omnipotent", d. h. können noch in die gewünschte Entwicklungsrichtung differenziert werden. Von embryonalen Stammzellen wird erwartet/erhofft, dass sie O rganund $\mathrm{G}$ ewebetransplantationen überflüssig machen, da das gewünschte Material nachwachsen soll. Aber auch adulte Stammzellen können möglicherweise in gewünschte Richtungen differenziert werden.

Heute sind vor allem hämatopoetische Stammzellen, gewonnen aus dem Knochenmark in der HämatoO nkologie in Anwendung: Autologe Stammzellentransplantationen haben in den letzten Jahren enorm zugenommen. Das Europäische Knochmarktransplantationsregister/ EBMT weist eine Zunahme von 2000 (autologer) Transplantationen Mitte der $90 \mathrm{er}$ Jahre auf 12.000 im Jahre 2000 und eine Zunahme von 2000 allogener (fremde Stammzellenspende) auf 4000 auf.

Nicht alle der medizinischen Anwendungen zeigten sich allerdings als gleich erfolgreich. Wie Assessments zur Hochdosischemotherapie nach Brustkrebs zeigten, war die Therapie in dieser Indikation wenig erfolgreich, aber wesentich teurer (NCCHTA 1998; ISEG 1999).

Während die öffentliche Diskussion sich ausschließlich auf den Diskurs zur „Ethik der Stammzellenforschung " beschränkt, wird nicht über den tatsächlichen medizinischen Nutzen geredet. Beispiele aus den weitverbreiteten hämatopoetischen Stammzellentransplantationen beweisen, dass nicht alle medizinischen Anwendungen sinnvoll sind. $\mathrm{Da}$ Stammzellentransplantationen aber sehr kostenintensive Interventionen sind, sind frühe Anwendungsbeobachtung und Evaluierung sowie eine öffentliche Nutzendiskussion vor breiter Anwendung unbedingt erforderlich. 


\section{Termine}

\section{5.-6. Dezember 2001 \\ Clinical Excellence in the NHS, \\ NICE \\ London}

http://www.nice.org.uk

14.-15. Dezember 2001

2. Berliner Intensivkurs "Evidence-Based Surgery“ im Unfallkrankenhaus Berlin dirk.stengel@ukb.de

18. Dezember 2001

Gesundheitsförderung gesunde Ernährung fördern Rathaus/Wien

21. Jänner 2002

Neue Aspekte zum Q ualitätsverbesserungsprozeß Die Rolle von Information und Kommunikation BMSG

9.-12. Juni 2002

$18^{\text {th }}$ Annual Meeting

ISTAHC/International Society of Technology Assessment in Health Care Berlin

http://www.istahc2002.de

30. J uni-5.J uli 2002

$18^{\text {th }}$ UICC International Cancer Congress

5. Juli: HTA in O ncology

O slo

http:// www.oslo2002.org
NCCHTA 1998: Bone marrow and peripheral blood stem cell transplantation for malignancy.

ISEG 1999, Erg. 2000: Hochdosischemotherapie mit autologer Stammzellentransplantation zur Therapie des metastasierenden Mammakarzinoms.

\section{Hyperbare Sauerstoff- therapie/HBO}

\section{Versorgung des Körpers mit Sauerstoff}

Die Hyperbare Oxygenierung ( $\mathrm{HBO}$ ) wird bei Erkrankungen eingesetzt, die auf Sauerstoffmangel, auf Keiminfektionen oder Gasblasen in der Blutstrombahn zurückgeführt werden. Während einer HBO wird bei einem Druck, der über dem normalen atmosphärischen Druck liegt, reiner Sauerstoff in einer Druckkammer eingeatmet. HBO wird häufig in Akutfällen wie Taucherunfall, Kohlenmonoxidvergiftung und in der Intensivmedizin angewandt.

In Deutschland wurde bereits 1994 die ambulante HBO -Anwendung aufgrund des fehlenden Nachweises des therapeutischen Nutzens als G KV-Kassenleistung nicht anerkannt. Eine erneute Evaluierung (1999/2000) überprüfte über 2600 wissenschaftliche Veröffentlichungen zur HBO, davon 12 Indikationen bei denen ein ambulanter Einsatz von HBO empfohlen wird. Weiters über 30 Indikationen, bei denen die HBO in klinischen Studien angewandt wurde.

Ergebnis: Trotz einer großen Anzahl von randomisierten klinischen Studien konnte die Wirksamkeit der HBO in den 30 evaluierten Indikationen nicht belegt werden. Aufgrund des weiterhin fehlenden Nachweises eines therapeutischen Nutzens (bei z. B. Hörsturz mit/ohne Tinnitus, DFS/Diabetisches Fußsyndrom, Knalltrauma etc.) wurde die Entscheidung von 1994 bestätigt und HBO nicht in den GKVLeistungskatalog aufgenommen. Weiters wurde als eindeutig experimentelle Indikation das Neuroblastom
Stadium IV eingestuft. Die Anwendung von HBO zur stationären Behandlung schwerer Krankheitsbilder wie Dekompressionskrankheit nach Tauchunfällen, Kohlenmonoxidvergiftungen etc. beruht nur auf Plausibilität und ist auch im Rahmen multidisziplinärer intensivmedizinischer Betreuung kein Therapiestandard. Weitere (internationale) Assessments bestätigen diese Ergebnisse.

Cochrane Review 2001: Hyperbaric oxygen for carbon monoxide poisoning.

KBV/BRD 2000: Hyperbare Sauerstofftherapie, http://www.kbv.de/hta.

DEC-Report/GB 2000: Hyperbaric oxygen therapy in the management of carbon monoxide poisoning, osteoradionecrosis, burns, skin grafts and crush injury, http://www.publichealth.bham.ac.uk/wmhtag/ g/ pdf/hyperbaric.pdf.

AETMIS/CA 2000: L'oxygénothérapie hyperbare au Q uébec,

http://www.aetmis.gouv.qc.ca/.

\section{Laufiende Assessments}

ICTAHC/Israel; MSAC/Australien.

\section{Sakrale Neuro- stimulation/SNS bei Inkontinenz}

\section{Implantation eines "Schrittmachers"}

Die Neurostimulation ist eine Methode, die - nach erfolglosen konservativen Therapieversuchen - bei Inkontinenz zum Einsatz kommt. Implantiert wird meist rückenmarknah ein kleiner Impulsgeber (Schrittmacher), der schwache elektrische Impulse an den für den Entleerungsvorgang zuständigen Sakralnerven abgibt.

Die Neurostimulation wird primär bei urinärer, aber auch bei Fäkalinkontinenz eingesetzt; sie bedarf eines stationären Eingriffs und ist sehr kostspielig.

Ergebnis: Etwa 50 \% jener Patienten, die nach erfolgloser konservativer Be- 


\section{Publikationen}

mulation behandelt wurden, waren nach 6-monatigem follow-up "trocken“. Bei etwa einem Drittel der behandelten Patienten funktioniert SNS gar nicht, bei einer großen Anzahl an Patienten (36\%) kommt es zu bedeutsamen Nebenwirkungen und Komplikationen, die wiederum medizinische Behandlung bedürfen. Da neben Unsicherheit in der medizinischen Wirksamkeit und Sicherheit, die Kosten-Nutzen Relation ungünstig ist, wurde in allen Ländern, in denen Evaluationen durchgeführt wurden, von einer (öffentlichen) Finanzierung außerhalb klinischer Studien abgeraten.

BSV/CH 2000: Sakralnerven-Stimulation zur Behandlung der Harninkontinenz.

CAHTA/SP 2000: Efficacy, effectiveness and safety of sacral neuromodulation in the treatment of urinary incontinence,

http://www.aatm.es/cgi-bin/frame.pl/ang/ pu.html.

MSAC/AU 2000: Sacral nerve stimulation for urinary incontinence,

http://www.health.gov.au/msac/pdfs/ msac1009.pdf.

\section{Laufende Assessments}

CCOHTA/CA: Sacral nerve devices for urinary incontinence.

\section{Spasmolytika: u. a. Botulinum toxin/Bt}

\section{Behandlung von Spastik}

Botulium toxin ist ein bakterielles Nervengift, das direkt in die betroffene Muskulatur injiziert wird. Bt ist in der Lage, die neuromuskuläre Übertragung zu blockieren und dadurch eine künstliche Lähmung herbeizuführen. Dieser Effekt macht sich therapeutisch zunutze, um z. B ausgeprägte Spastik zu lösen.

Spasmolytika (sowohl Botulinum toxin, wie Baclofen, Tizanidine etc.) kommen bei Multipler Sklerose, Zerebralparese bei Kinder und auch nach Schlaganfall zum Einsatz.
Ergebnis: Die beiden vorliegenden Cochrane Reviews geben keine Empfehlungen zugunsten einer Verschreibung von Spasmolytika und Botulinum toxin $a b$ : Aufgrund der unterschiedlichsten Messmethoden bei Spastik und damit verbunden der funktionellen Bewertung der Wirksamkeit von Spasmolytika sind die Ergebnisse der vorliegenden Studien nicht überzeugend (weder unterstützend noch widerlegend). Dringend erforderlich sind gültigen Messmethoden für Spastik. Der Einsatz von Bt wird aber zunehmend als Alternative oder als Ergänzung zu herkömmlichen Therapien akzeptiert: Vor einer Verbreitung sollten aber weitere Forschungen zu Langzeiteffekt, Dosierung und Toxinverteilung abgewartet werden.

Cochrane Review 2001: Botulinum toxin type $A$ in the treatment of lower limb spasticity in cerebral palsy.

Cochrane Review 2001: Anti-spasticity agents for multiple sclerosis.

\section{Laufende Assessments}

CCOHTA/CA: Efficacy of botulinim toxin for treatment of spasticity.

Cochrane Protocol: Botulinum toxin for spasticity after stroke or non-progressive

NCCHTA/GB: The management of pain and spasticity in multiple sclerosis.

\section{Alzheimer- Medikamente}

\section{Donepezil, Rivastigmine, Galantamine, Metrifonte}

Etwa $6 \%$ der Bevölkerung über 60 Jahre erkranken an Alzheimer: Die Ursachen sind noch ungeklärt. Die medikamentöse Therapie zielt auf eine zeitliche Verzögerung des Krankheitsverlaufes $a b$. Von medikamentöser Behandlung wird erwartet, dass sie in der frühen und mittleren Krankheitsphase eine Verbesserung der Lebensqualität bewirken. brain lesion.

\section{NCCHTA/GB:}

http://www.hta.nhsweb.nhs.uk/

Systematic reviews of wound care management:

(1) A rapid and systematic review of the clinical effectiveness and costeffectiveness of debriding agents in treating surgical wounds healing by secondary intention, 2001

(2) Dressings and topical agents used in the healing of chronic wounds, 1999

(3) Systematic reviews of wound care management: antimicrobial agents for chronic wounds; diabetic foot ulceration, 2000

(4) beds; compression; laser therapy, therapeutic ultrasound, electrotherapy and electromagnetic therapy, 2001

Systematic review of treatments for severe psoriasis, 2000.

Screening for hypercholesterolaemia versus case finding for familial hypercholesterolaemia: a systematic review and costeffectiveness analysis, 2000

Comparison of the effectiveness of inhaler devices in asthma and chronic obstructive airways disease: a systematic review of the literature, 2001

The cost-effectiveness of magnetic resonance imaging for investigation of the knee joint, 2001

A rapid and systematic review of the clinical effectiveness and cost-effectiveness of gemcitabine for the treatment of pancreatic cancer, 2001

A rapid and systematic review of the clinical effectiveness and cost-effectiveness of topotecan for ovarian cancer, 2001 
HTA-Projekte am ITA

Medizinische Effektivität von Ilb/IIla-Rezeptorantagonisten

in der Therapie von akuten Koronarsyndromen

1.10.01-31.12.01 Projektleitung:

C. Wild, W. Frank

Genetische Diagnostik bei hereditären Krebserkrankungen,

insb. bei Mamma- und bei

Kolonkarzinom

1.10.01-31.3.02

Projektleitung: S. Jonas

\section{HTA-Vorträge} am ITA

Paul Krabbe, PhD

Gesundheitsökonom

Universität Nijmegen/N L

Dept. Medical TA

„Cost-Effectiveness Ratios

for Resource Rationing in

Health Care Systems"

Montag 10. Dez. 2001

10:00-12:00

Walter Peissl, Dr. ITA/Ö AW

„Patientenkarten: Eine Technikfolgen-Abschätzung“

Montag 28. Jänner 2002 10:00-12:00

Impressum

Redaktion: Claudia Wild Saskia Schlichting

Graphik: Manuela Kaitna Eigentümer und Herausgeber: ITA - Institut für Technikfolgen-

Abschätzung der Ö sterreichischen Akademike der Wissenschaften,

A-1030 Wien, Strohgasse 45/3

http://www.oeaw.ac.at/ita

Der HTA-N ewsletter erscheint $10 \times$ pro Jahr und ausschließlich auf der ITA-homepage ISSN : 1680-9602
Es liegen zahlreiche systematische Reviews und randomisierte Studien zu den einzelnen Medikamenten vor: Wenngleich die therapeutischen Ansätze der Alzheimer Medikamente unterschiedlich sind, wurden die klinischen Studien in zwei Assessments nach klinischer Wirksamkeit der Medikamente, Einfluß auf Lebensqualität und Kosten-Nuten bei leichtem bis mäßigem Alzheimer evaluiert.

Ergebnis: Trot geringer Differenzen der Wirksamkeit (von Donepezil, Metrifonate, Rivastigmine, Galantamine) konnte keine Therapie eine Progression verhindern. Sie zeigten auch in den meisten Fällen nur moderate Verbesserungen auf verschiedenen (kognitiven, funktionalen, Verhaltens-)Bewertungsskalen.

O ffen bleibt, ob moderate Veränderungen auf den klinischen Skalen einer realen Veränderung der Bewältigung des täglichen Lebens gleichkommt. Es werden zwar Kostenersparnisse durch geringere Inanspruchnahme von mobiler/ambulanter Pflege und Hausbetreuung erhofft, die vorliegenden Kosten-Nutzen Evaluationen bestätigen diese Hoffnungen aber nicht (weder einsparend noch erhöhend).

Wenngleich der Nachweis eines signifikanten $\mathrm{N}$ utens nicht vorliegt, gibt NICE/G B Anleitung zu bedingtem/restriktivem Einsatz der Medikamente.

DEC Report $1997 \&$ 1998: Donepezil in the treatment of mild to moderate senile dementia of the Alzheimer type (SDAT) \& Rivastigmine (Exelon TM) in the treatment of senile dementia of the Alzheimer type (SDAT), http://www.doh.gov.uk/research/ swro/rd/publicat/dec/.

ICTAHC/Israel1999: Pharmacological treatment of Alzheimer's Disease: clinical and economical aspects.

CCOHTA/CA 2000: Drug treatments for Alzheimer's disease. I (A comparative analysis of clinical trials), II (A review of outcome measures in clinical trials), III (A review of pharmacoeconomic evaluations).

CCOHTA/CA 2001: Drug treatments for Alzheimer's Disease: efficacy, outcome measurements and cost-effectiveness, http://www.ccohta.ca/newweb/pubapp/ pubsearch.asp\#\#.
NICE/GB 2001: Guidance on the use of donepezil, rivastigmine and galantamine for the treatment of Alzheimer's Disease, http://www.nice.org.uk/pdf/Alzheimer hta.pdf.

\section{Laufiende Assessments}

CCOHTA/CA: New drugs for Alzheimer's disease - systematic review.

\section{HTA-Suchhilfe}

\section{HTA-Linkliste auch als Rechercheseminar}

"Recherche-Hilfe HTA“ ist eine interaktive Linksammlung und ein Suchinstrument, welches durch die HTAEinheit des Instituts für Technikfolgen-Abschätzung (ITA) zusammengestellt wurde (und regelmäßig überarbeitet wird).

Sie ist als Unterstützung für (rasche) Recherchen in der Gesundheitsadministration, aber auch in der klinischen Medizin gedacht. Auf Anregung des BMSG/VIII wurde auch ein Unterrichtsmodul entwickelt, in dem Recherchetechniken mithilfe der HTALinkliste gelehrt/geübt werden. Bei Interesse kann ein mehrstündiges Recherche-Seminar gegen Honorar "angefordert" werden.

http://www.oeaw.ac.at/ita/htalinks.htm.

\section{Vorausschau Februar}

- Invasive Aspergillose

- Akupunktur

- Clopidogrel

- Polysomnographie

- Kolorektalscreening.

Wir freuen uns über Reaktionen und Hinweise insbes. auf für Österreich relevante Themen!!

Kommunkation an: cwild@oeaw.ac.at 